\title{
Aging and induced pluripotent stem cells
}

\author{
Abbreviations: SNPs, single nucleotide polymorphisms; \\ DNA, deoxyribonucleic acid; iPSCs, induced pluripotent stem cells; \\ HSCs, hematopoietic stem cells
}

\section{Editorial}

Aging in humans is irreversible, characterized by a decline of physiological functions in various organs and tissues, leading to multidimensional process of physical, psychological, and social change, causing most human diseases and an increasing probability of death. At present, the exact mechanism and biological basis of aging is unknown. The antagonistic pleiotropy theory and competing model of senescence explain the aging occurrence of some physiological functions of human body from different angles. The single nucleotide polymorphisms (SNPs) of genome suggest the individual's difference of aging process is associated with SNPsrelated individual's sensitivity to diseases. As the naturally occurring DNA damages and age-associated accumulation of DNA damage as well as decline in gene expression regulation indicate individual life expectancy, whereas the shortening mechanism of telomeric terminals of chromosomes and Hayflick limit of cell division determine human life span.

The genomic aging exerts impact on various physiological functions, also imprints individual's appearance, such as wrinkled, thinning skin, graying and loss of hair. Feeling helpless before slowing down senescence of the genetic materials people turn to antiaging products, spending billions of dollars each year on such creams and lotions for decreasing signs of aging and nutrients for postponing decline of physiological functions. Indeed, recently, scientists carried out reversing muscle aging in mice model through feeding an experimental compound and made a comment that for humans the effect would be similar to a 60 year-old feeling like a 20 year-old, but still powerless for reversing of genomic aging, even a little. Is there a possibility of U-turn in the highway of life progress? Recently, the discovery that mature cells can be reprogrammed to become pluripotent and the development of rehabilitative and regenerative medicine rekindle spark in hope of humans against aging. The induced pluripotent stem cells (iPSCs) hold great promise in the field of regenerative medicine because each individual could have their own pluripotent stem cell line and these unlimited supplies of autologous cells bypass body's immune defense system, immune rejection, this will be a new milestone in transplantation medicine, one of the most challenging and complex areas of modern medicine. Nowadays, iPSCs are mainly used in medical research, including organ synthesis, disease modeling of cellular basis and drug development, tissue repair in animal model.

Here is an opinion regarding the possibility and feasibility of iPSCs application in gerontology. The gerontology is the study of the social, psychological and biological aspects of aging, itself is a youthful field, its progress having been slowed by aging's daunting complexity and a tendency among scientists and doctors to consider diseases as entirely separate, rather than as manifestations of a common origin. In comparison to the complexity and difficulty of synthesizing other

\author{
Volume I Issue 2 - 2014 \\ Yunlong Qin \\ Department of Obstetrician/Gynecologist, Morehouse School \\ of Medicine, USA
}

Correspondence: Yunlong Qin, Department of Obstetrician/ Gynecologist, Morehouse School of Medicine, Cancer Biology Program, 80 Jesse Hill Jr, Dr, Atlanta, GA 30303-303I, Complex Biological Systems Alliance, USA, Tel 470248I359, Emailyqin05@yahoo.com

Received: October 09, 2014 | Published: October 20, 2014

organs or systems through iPSCs, there is great advantage for iPSCs being applied into the immune system, the functions of which are mainly carried out by immune cells, same as red blood cells, these immune cells travel to everywhere of human body. The application of cell levels of iPSCs is more reliable and easier in technology. The aging process accompanies gradual deterioration of the immune system. This age-associated immune deficiency is ubiquitous, decreasing host's capacity to respond to infections, increasing individual's sensitivity to most human diseases, including autoimmune disorders. The functional capacity of T-cells is most influenced by the effects of aging. Since all the blood cells come from pluripotent hematopoietic stem cells (HSCs) of bone marrow, no matter what age you are, but these freshly produced immune cells from aging body are still senescent. The raised question is when is best time period to collect adult cells to be reprogrammed genetically into iPSCs? Here is a suggestion with the best timing for preparing the individual specific iPSCs should be a period of time of strongest function of immune system, thinking roughly 30 years of age. Comparing to the cord blood stem cells, unlimited supplies of iPSCs will have more attractive application prospect once its safety is more reliable and accepted and more mature in technology. There is an expectation to help elderly people re-establish a strong immune system to resist the attack of pathogens including bacteria and viruses and make them live in a higher level of health state. Interestingly, recently, type O red blood cells were synthesized at the Scottish National Blood Transfusion Service from iPSCs. Type O can be transfused into all patients this is unprecedented brilliance in the transfusion history considering more than 100million blood donations are collected globally every year. Where do we go on the journey against aging? It seems we see some light we could reach from the rapid progress of life sciences and biotechnology today. Hopefully, it is not star light.

\section{Acknowledgements}

None.

\section{Conflict of interest}

Author declares that there is no conflict of interest. 